\title{
Funcionamiento de los fondos de inversión en El Salvador
}

\author{
Entrevista a Francisco Santa Cruz Pacheco \\ Gerente general de la Gestora de Fondos de Inversión Banagrícola S.A. \\ Licenciado en Administrador de Empresas de la UCA \\ Maestría en Dirección Financiera de la Universidad Complutense de Madrid
}

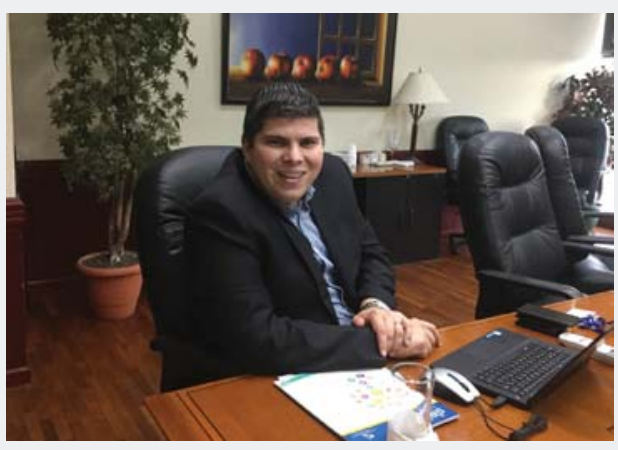

Qué es un fondo de inversión?

Un fondo de inversión es el conjunto de aportes de diversos inversionistas, a quien la norma los denomina aportes como participes de ese fondo; ese conjunto que se reúne es administrado por una sociedad gestora y son invertidos los recursos a cuenta y riesgo del inversionista.

\begin{abstract}
¿Por qué usar los fondos de inversión?
Si yo, individualmente, quisiera ir al mercado de valores sería muy difícil que pueda participar en algún título del mercado que me interese porque estos títulos generalmente tienen rangos específicos (montos) con los cuales yo tengo que invertir y de repente la inversión que yo deseo realizar no están grande o no se sujeta al monto que está fijado en esa transacción. Entonces a través de un fondo, se reúne el capital de un cierto número de personas, con los cuales se genera un patrimonio bastante considerable, que logra un poder de mayor de negociación en la bolsa, por lo que la sociedad gestora puede ir y adquirir esos títulos que yo, individualmente, no podría hacerlo.
\end{abstract}

\section{¿Cómo funciona un fondo de inversión?}

Primero definamos algunos términos ya que es importante que nos empecemos como a familiarizar con ellos.

La figura de inversión se llama aporte y cuando hay un retiro se llama rescate.

El conjunto de aportes de los diferentes inversionistas se llaman participes y el capital es invertidos de acuerdo a la política de inversión y es administrado por la gestora a cuenta y riesgo de un inversionista.

Supongamos si yo invierto en un fondo o participo en un fondo a cuenta y riesgo mio, porque la gestora me va a explicar a mi, el reglamento del fondo, la política de inversión, los riesgos que tiene el invertir en el fondo y yo como inversionista voy a quedar claro en que estoy invirtiendo y el riesgo que estoy asumiendo, así como los beneficios que pueden haber en un fondo de inversión.

Los inversionistas se reúnen tienen un objetivo común, ven un fondo que esté en el mercado, van varios y depositan su dinero en la gestora, ya que la gestora es quien administra el fondo, entonces vienen los inversionistas y depositan el dinero en el fondo. Cada fondo tienen distintas políticas de inversión, dependiendo de la política de inversión que adopte, es el nivel de riesgo que tiene el fondo y eso es lo que se evalúa en el momento que se hace una calificación de riesgo del fondo, se ve en qué invierte o cuál es su política de inversión, cuales son los activos en los que él tiene posesión.

La gestora, de acuerdo a la política de inversión del fondo, invierte.

Los resultados o la rentabilidad que se obtiene de esa inversión es repartida entre todos los inversionistas que participan dentro del fondo. Aquí es similar al tema de una acción, cuando yo participo en comprar una acción de una empresa, yo soy dueño de una parte de esa empresa, en el fondo de inversión es lo mismo, yo voy a ser un participe del fondo y voy a ser dueño de una cuota, que se llama cuota de participación.

\section{¿Quién regula el funcionamiento de las gestoras de fondos de inversión?}

La gestora es una entidad financiera como lo es un banco, una sociedad de ahorro y crédito, de igual manera está regulada y supervisada por la Superintendencia del Sistema Financiero, eso da cierta seguridad a todo este esquema.

Asimismo, recibimos igual que un banco, diferentes auditorias en todos los procesos, sistemas, porque eso da cierta tranquilidad a los usuarios que nosotros estamos trabajando bajo los estándares que las normas locales permiten.

La Ley de Fondos de Inversión es la base de todo el proceso y de ahí surgen varias normativas las cuales son alrededor de 13 que ha dictado el Banco Central de Reserva sobre diversos puntos, entre los que están: la constitución, los requisitos legales que debe de cumplir una gestora para poder constituirse, el inicio de operación de una gestora, la constitución de un fondo de inversión; qué requisitos debe de cumplir un fondo para constituirse, quienes va a comercializar con los fondos porque son una figura distinta que existe dentro de todo este funcionamiento, quien comercializa los fondos son las entidades comercializadoras autorizadas por la Superintendencia del Sistema Financiero.

\section{“La Ley de Fondos de inversión es la base de todo el proceso y de ahí surgen varias normativas las cuales son alrededor de 13 normativas que ha dictado el Banco Central de Reserva..."}




\section{¿Qué beneficios reciben las personas que invierten en fondos de inversión frente alguien que hace un depósito a plazo fijo?}

Los beneficios son muchos iniciando que estamos acostumbrados a los productos tradicionales de inversión como una cuenta de ahorro, un depósito a plazo. En estos tiempos, en otros mercados hay muchas figuras de inversión que le permite diversificar y acceder a otro tipo de portafolios.

Por ejemplo deposito mis ahorros en un depósito a plazos fijo estoy concentrando mi riesgo, el riesgo que estoy asumiendo es el que tiene el banco. En caso de quiebra un banco en el instituto de garantía del depósito hay un monto por el cual se responde por un depósito a plazo, pero no responde por todo mi capital y yo pueda perder parte de él.

En cambio, en un fondo de inversión no se va invertir en solo una institución financiera sino que en varias, en la bolsa de valores, en el mercado local e internacional, entonces estoy diversificando el riesgo. En cambio, ocurriese una pérdida en el fondo que se tiene invertido en cierta posición en esta institución la pérdida va a ser menor porque el fondo va a tener otras posiciones en otros bancos que no va ser la única en esta institución, entonces como inversionista estoy diversificando el riesgo, esa es una primer ganancia o un primer beneficio de porque invertir en un fondo de inversiones.

Un segundo beneficio es que el personal con el que trabajan las gestoras son expertos que están invirtiendo por mí, su día a día va a ser el invertir o buscar las opciones mejores opciones para invertir el dinero de los inversionistas.

La norma que regula o estipula que la persona que invierte es el administrador de inversiones del fondo, esta persona también tiene una autorización especial para poder hacer su labor, es bien importante porque esa persona ha recibido esa autorización y tiene que cumplir ciertos requisitos para ser nombrado un administrador de inversiones, ahí tú te das cuenta que hay expertos que han cumplido ciertos requisitos que están invirtiendo el dinero por ti, o sea que ya tienen cierta experiencia en el mercado y conocen un poco donde pueden ir invirtiendo de acuerdo las políticas de inversiones del fondo.

Estos expertos están invirtiendo en nombre del inversionista, son los encargados de vigilar, ver y analizar todo en estas instituciones en las que se va a invertir. Por ejemplo, en el reglamento de un fondo de inversión, se establece que el administrador de inversiones puede invertir en instituciones financieras que tengan calificación den riesgo, por ejemplo triple $B$, entonces yo no puedo invertir sino es triple B. El administrador de fondos tiene que hacer su análisis y decir que el banco tiene esta categoría de riesgo entonces puedo invertir en él. El análisis que realizan los expertos se sustenta porque hacen determinadas inversiones y eso da mayor tranquilidad a los inversionistas porque el experto que está invirtiendo tiene cierto análisis, cierto conocimiento en lo que está realizando.

Por ejemplo, si voy a abrir un deposito a plazos en un banco, a veces porque me ofrecen la mejor tasa, pero yo como inversionista, no hago un análisis financiero de que este banco tiene cierta calificación de riesgo o no me pregunto cómo va su volumen de crecimiento de depósito o de cartera, entonces a veces, el inversionista, entonces tomo una decisión en base a que la tasa es buena, allí lo invierto pero no veo los riesgos que podrías asumir por no hacer cierto análisis. A veces, como inversionista no indago la calificación de riesgo del banco en donde estoy abriendo el depósito.

Un tercer beneficio son las economías de escala y el poder de negociación. Como decía, yo solo individualmente es bien difícil ir y acceder a cierto instrumento financiero o de valores que el fondo si va a poder hacer, por ejemplo, abrir un deposito a plazos de $\$ 5000$ dólares no es lo $\mathrm{m}$ ismo que abrir un deposito a plazos de $\$ 50$ millones de dólares, con éste último obtengo poder de negociación; de negociar la tasa con el banco o con la institución financiera decirle mira yo te voy a dar cierto fondos, qué tasa me ofreces, se puede hablar con varias instituciones y buscar que dentro del riesgo que yo acepto o tolero quien me ofrece las mejor condiciones para poder invertir en ese banco, personalmente puedo hacerlo dentro de mis capacidades puedo invertir $\$ 5000$ dólares y los voy a invertir de mejor manera pero el fondo gana un mayor poder de negociación.

Economías de escalas porque los gastos que tenga el fondo se distribuyen entre todos los participantes del fondo, entonces que es lo que cobran las gestoras, hay una comisión por la administración de los fondos. Esa comisión está realizada para que se cobre a cada uno de los partícipes pero no es lo mismo que yo individualmente contrate a alguien experto para que realce todo este andamiaje, entonces existen economías de escalas que me permiten los fondos.

Un cuarto beneficio es el tema de la rentabilidad obviamente como resultado de la mezcla de activos y renta fija o variable que haga el fondo, lo que está buscando es rentabilidad, como decíamos de una cuenta de ahorro, de un deposito a plazos, va a depender un poco de los tiempos y de los riesgos que asuma el fondo. Puede haber un fondo, por ejemplo, de corta duración o de corto plazo que tal vez no gane mucho, no gane igual a uno de mediano plazo, también un fondo de mayor riesgo, hay diversos tipos de fondos que pueden hacerse dependiendo en que invierten, entonces así asumen el riesgo, puede ser un fondo que invierta en títulos a mayor de un año, el riesgo es un poco mayor pero el rendimiento al final es mejor que si invierten en corto plazo. 
Un quinto beneficio es la liquidez, un fondo de corto plazo tal vez no gana tanta rentabilidad aunque es mejor y más atractiva que la de un depósito pero la liquidez es más rápida. Puede ser que en la mañana yo este solicitando rescate de mis cuotas de participación y en la misma tarde la gestora me lo esté depositando mientras yo decida que voy a recibir ese pago. Entonces, la liquidez inmediata de mi transacción, como por ejemplo un depósito a plazos ya sea que la tenga o no a un nivel más caro no existe una penalización, dependiendo del fondo se llama "Pactos de Permanencia", cuando yo tengo cierto pacto entre el inversionista y la gestora en el que digo por este periodo de tiempo no puedo salir del fondo; hay dos tipos de fondo, abiertos y cerrados; los abiertos son los que podes entrar y salir y no tienen un pacto de permanencia, podes salir o entrar en cualquier momento y los cerrados son los que tienen una fecha estipulada en la que se puede salir del fondo. Entonces, obtiene esa liquidez inmediata.

Un sexto beneficio seria la seguridad de la inversión, dado las regulaciones que se tienen y los altos estándares de desempeño y conducta a cumplir, da una mayor seguridad a los usuarios. Para la comercialización de los fondo de inversión es importante informar al inversionista en que se está invirtiendo y le explico claramente mi política de inversiones.

En un fondo de corto plazo, la volatilidad es menor porque estas invirtiendo en títulos bastante seguros, entonces generalmente los títulos que son más volátiles son más a largo plazo, se vuelve un poco más seguro en ese sentido porque estás invirtiendo en algo que estás viendo.

\section{¿Qué sucede si la gestora o el administrador de inversiones incumplen la política de inversiones?}

Si una gestora indica que va a invertir en fondos que son triple $A$, por ejemplo, pero viene el administrador de inversiones y dice no esta institución financiera $X$ me está pagando una rentabilidad que me va a mejorar la rentabilidad del fondo y yo voy a invertir allí, y luego invirtió en una institución que es triple $B$, cuando la política le demandaba solo invertir en triple A. Entonces él está incumpliendo la política, si bien es cierto, la razón es para buscar rentabilidad pero está incumpliendo la política, entonces si el inversionista pierde sí podría demandar a la gestora ante la Superintendencia del Sistema Financiero, que es quien nos regula y decir que él tiene un daño y perjuicio porque no está invirtiendo en lo que a él se le dijo o en lo que el acepto que se iba a invertir. Entonces, la gestora tiene la obligación de constituir una fianza una garantía hasta por el $1 \%$ del patrimonio que administre, la menor es de $\$ 500$ mil dólares, o sea desde $\$ 500$ mil dólares para arriba dependiendo del patrimonio de los fondos que yo administro, constituye una garantía y esa garantía en primer momento responde que se haga uso de la Superintendencia del Sistema Financiero.
Si eso no es suficiente y se evidencia que hay una mala fe de la gestora o un incumplimiento, incluso la gestora responde con su propio patrimonio. Para llegar a ser una gestora se constituye con un patrimonio mínimo que la ley manda y son $\$ 500 \mathrm{mil}$ dólares, de tal manera que es un millón de dólares como mínimo el que responde ante cualquier hecho de mala fe.

\section{¿Quiénes pueden invertir en un fondo de inversión?}

Todos pueden invertir por lo que no hay ninguna limitante, cualquier persona natural o jurídica que cumpla los filtros de cumplimiento que hoy en día hay como lo es la Ley Contra el Lavado de Dinero y de Activos y su Reglamento, que son los controles que como gestora debemos de hacer, la procedencia de donde viene el dinero, es lo que se revisa. Una vez pasa esos filtros de cumplimiento cualquier persona natural o jurídica puede invertir en el fondo.

No existe limitante de edad, pero si existen ciertos requisitos legales de documentación que se solicitan que tal vez una persona menor de edad no cumpla, entonces podría decirse que todo mayor de edad puede participar en un fondo porque ya tiene su Documento único de Identidad (DUI), su Número de Identificación Tributaria (NIT) que son los documentos que se solicitan y de ahí se apertura una cuenta de fondo en la cual desee invertir y puede participar en el fondo de inversión.

\section{¿Cómo se determina el grado de riesgo del inversionista?}

Cuando una gestora está vendiendo un fondo de inversión, asesorando a un inversionista lo primero que hace es determinar el perfil de riesgo del inversionista, para saber si él es: conservador, moderado o agresivo.

El conservador es el que menos quiere perder, entonces ese quiere invertir en inversiones seguras, por lo que en el conservador podemos participar todos.

Hay perfiles de inversionistas que quiere arriesgar más, par ganar más entre ellos encontramos a los inversionistas con perfil moderado y agresivo solo si determinamos que el cliente tiene ese perfil moderado o agresivo o si se determina que el cliente es conservador pero quiere invertir en un fondo agresivo, se va a firmar una declaración en donde se acepta invertir en un fondo que no es de tu perfil, para que el inversionista ahí se eduque y entienda que esta invirtiendo en algo que por su perfil no debería. El que asume el riesgo es el inversionista, entonces es bien importante, como Gestora Banagrícola que eduquemos y expliquemos bien a nuestros participes sobre todos los beneficios, como todos los riesgos que pueden haber para que la persona este lo mejor educada y conozca del tema. 


\section{¿Cuántos fondos de inversión hay en El Salvador?}

Hay tres gestoras que están buscando la aprobación de estos fondos. Actualmente solo hay una de I as tres que tiene su fondo ya funcionando. Las otras dos todavía están en el proceso de autorización del fondo.

Las dos gestoras ya autorizadas, como administradoras de fondos de administración aun no cuentan con un producto autorizado.

Por lo que, actualmente en circulación a nivel nacional solo hay un fondo de inversión que es el Fondo de Inversión Servicios Generales Bursátiles, que nació en octubre del 2016, y es un fondo de corto plazo de liquidez abierto que busca igual rentabilidad y liquidez.

\section{¿Qué sucede si al fallecer el inversionista tiene un programa de beneficiaros?}

El documento legal que se va a firmar al participar o abrir una cuenta de fondos de inversión es el contrato de suscripción de cuotas, de participación del fondo, entonces en este contrato se delegan beneficiarios en, y al fallecer se les entregan las cuotas de participación, el beneficiario decide si continua invirtiendo en el fondo o va hacer un rescate total de las cuotas de participación que hasta ese momento tenía y retira el dinero invertido que tenía la persona que lo había designado como beneficiario. En este tema, si es igual al depósito a plazos, se puede o no designar beneficiarios porque existe también la opción de que él que no quiera designar a nadie, no por eso no puede ser parte del fondo, pero si está la posibilidad para que pueda designar beneficiarios.

\section{¿Cuánto seria el porcentaje de rentabilidad que se espera dejen los fondos de inversión?}

Depende mucho de los tiempos por ejemplo en un depósito a plazos depende la tasa, si lo invierto a 30 , 60,180 días, entre mayor sea el tiempo de plazo mayor es la tasa que paga un depósito a plazo.

En cambio en un fondo de inversión va a depender del tipo de fondo, si el fondo es de corto plazo, se puede comparar con un depósito a plazo de menor plazo en el tiempo, ahí van haciendo las comparaciones, qué vamos a buscar nosotros porque no hay nada matemático que diga si yo invertís en un depósito tu tasa es de $3 \%$ y si se invierte en el fondo tu tasa es de $3.5 \%$, no hay nada así escrito.

Fondo de Inversión de Banagrícola

\section{¿En qué tiempo se inició la gestora Banagrícola?}

La gestora Banagrícola viene desde finales de 2015 constituyéndose, se recibió la autorización de constitución legal en mayo de 2016, luego la siguiente autorización fue la autorización de inicio de operaciones, esa donde te revisan todo el sistema que se va a ocupar y todos los procedimientos que a seguir, esa se recibió en mayo de 2017. Luego se autoriza al administrador de inversiones, esta persona fue autorizada por la Superintendencia en junio de 2017. Posteriormente vienen los comercializadores que son el Banco Agrícola y Valores Banagrícola, que ya están autorizados por la Superintendencia, esa fue recibida en julio de 2017 y la última que se esta esperando es la del fondo de inversión que esperamos recibirla en septiembre de 2017, para poder ya salir al mercado, en octubre 2017. Ya tenemos todos los procesos revisados y aprobados por la Superintendencia.

\section{¿Qué esperan ofrecer al mercado con el fondo de inversión?}

Se procura ser lo más competitivo posible, posicionarnos en ser una mejor opción que los instrumentos de ahorro que ya existen y obviamente el beneficio mayor de fondo es la liquidez, al final buscas ser más rentable que estas figuras tradicionales y al mismo tiempo estas dando una liquidez inmediata.

Por qué hablo mucho de esa liquidez, porque suele pasar que yo tengo ahorrada cierta cantidad, el día de mañana me surge una emergencia, una necesidad y yo necesito de $X$ cantidad de dinero, en el fondo la puedo retirar, digamos que no es todo lo que retiro sino que dejo una parte, esa parte que dejo sigue ganando la misma rentabilidad que ganaba anteriormente o la obtuviste el mismo día o a más tardar el siguiente, por ejemplo en un depósito a plazo no funciona así de esa forma, entonces es bien importante que el inversionista en este primer fondo que vamos a formar evalué esas dos características; la rentabilidad que va a obtener y la liquidez inmediata que va a tener de los fondos que tiene invertidos.

\section{¿Cuál es la política de inversión que seguirá Banagricola?}

La política de inversión porque se busca es darle mayor de seguridad a los clientes. El grupo de empresas de la que somos miembros, siempre tiene cuidado en donde está invirtiendo, el riesgo del tercero que está invirtiendo, por lo que el banco siempre cuida la reputación y en este primero fondo que el fondo es conservador de corto plazo, que no es un fondo agresivo, está cuidando los emisores en los que está invirtiendo.

\section{¿El enfoque de ustedes es más a la seguridad del inversionista?}

Obviamente si, nosotros no nos sentiríamos cómodos invirtiendo en ciertas instituciones que tienen cierta calificación de riesgo porque estamos cuidando la seguridad del inversionista. 


\section{¿Cuál es la ventaja competitiva que ofrecen a los inversionistas?}

Nos apalancamos en toda la organización del banco. El banco tiene un área especializada de riesgos, que diariamente está evaluando a todos los emisores que hay en la plaza a nivel nacional. Por tanto. si yo quiero invertir en un determinado emisor, tengo a disposición un análisis de un especialista en el que me dice los riesgos que hay y lo comparo con mi política de inversiones y ya digo que puedo invertir o no. A la hora de comparar los distintos fondo de inversión en el mercado, podrá parecer que son iguales, pero esto hace la diferencia, todo el andamiaje de la gestora y así poder tener mayor seguridad a los clientes.

Actualmente la gestora que ya está operando en el mercado es calificación triple B+ entonces eso ya nos dices más o menos en que invierte el fondo de inversión. Por lo que hay que conocer qué calificación tiene un fondo de inversión, para identificar el riesgo que se está asumiendo, aunado a esto diferenciarnos de los productos tradicionales.

\section{¿Ustedes han pensado en incorporar a los inversionistas en los programas de formación o de sensibilización de estos procesos?}

Al estar preparada la normativa se quiere que además de comercializar el producto, hacer una campaña de educación financiera en el tema de fondos de inversión, para explicarle bien al cliente.

Cuando hicimos los estudios de mercado para determinar si salíamos o no en este proyecto se observó que a veces figura la anterior administración de cartera u otras figuras no todos los inversionistas las entienden para saber en qué se está invirtiendo, por lo que muchos pensaban que el banco respondía sobre su dinero.

A raíz de ello, se ha preparado, material educativo explicando la figura del fondo de inversión. Es importante que los inversionistas comprendan lo que gana al igual que los riesgos que asume al participar en un fondo de inversión, entonces como digo hay fondos dependiendo el perfil hay fondos para todas las personas pero tenemos que estar claros y saber que riesgos estoy asumiendo

\section{¿Cuál es el objetivo que tienen ustedes como institución, fortalecer la inversión o también fortalecer a los emisores o generar una dualidad en este proceso?}

Es una combinación de ambas cosas porque a veces había emisores o dejaban de participar ciertas personas como emisores del mercado de valores, no había inversionistas. Aquí se habla mucho que si las AFP no compraban emisiones, no tienes un inversionista que participe en ellos.

En los fondos de inversión se abre más la cantidad de participantes o inversionistas que van a poder participar, en emisiones de distintos tipos, que estén bajo la política de inversión, pero se abre esa posibilidad de que existan más inversionistas que antes no los había.

Aquí nos vamos a los tradicionales AFP, bancos que invertían y si ellos no invierten no había quien te comprará tu emisión y eso pudimos verlo con el tema de la titularizaciones que en un inicio se vendían pero después cuando fueron apareciendo más participantes costaba más vender las emisiones, es igual con las emisiones que hay de los diferentes grupos, de repente cuesta más venderlas porque te piden que tengas alrededor $\$ 10$ mil dólares para invertir y para entrar, entonces a veces no todos los inversionistas tiene esa disposición para entrar.

Con el fondo si va a poder entrar ya qué buscamos siempre respetar la política de inversión para darle la seguridad al inversionista, poder ser una opción más de participar con un determinado emisor.

\section{¿Cómo buscan ustedes contribuir de alguna manera a la economía del país con la creación de los fondos?}

Creo que es importante el desarrollo educativo que va a tener, el paso que vamos a dar es bien importante porque estamos dejando atrás las opciones tradicionales y estamos subiendo a una opción distinta o novedosa. En esa parte nosotros si nos hemos tomado muy en serio la parte de hacer un componente educativo para toda la población que entienda los fondos de inversión.

En segundo lugar hay muchos proyectos de nación, proyectos de empresas pequeñas, medianas que se pueden desarrollar a través de un fondo, puede nacer crearse, evolucionar o ser la fuente financiera de determinado proyecto.

También en el tema de las pensiones hay mucho conflicto, y las personas se hacen preguntas ¿Cuándo me jubile la pensión que voy a tener no me va alcanzar para nada?, por lo que un fondo de inversión puede volverse una alternativa que si yo ahorro $X$ cantidad de dinero me asegura que aquí a 10 años voy a tener cierta cantidad de retribución, entonces en base de esa necesidad creyendo que es una necesidad del mercado local, estamos ideando un fondo de ese tipo.

Debido a lo anterior, es importante que esa visión de armar o desarrollar esos instrumentos financieros que venga a ayudar, ya sea a una persona o un proyecto determinado que exista en el mercado local, creo que ese es nuestro ideal y hay muchos ejemplos en la región, no solo en Centroamérica sino que en Latinoamérica de fondos de inversión que a través de ellos se ha desarrollado proyectos y no habían inversionistas que creían en ese proyecto, hay entidades del propio banco que no invierten en ciertas cosas porque no quiere poner en riesgo el capital de los accionistas del banco pero en este caso si los inversionistas o los partícipes toleran el riesgo en ese proyecto bien se puede realizar. 


\section{E N T R E V I S T A FUNCIONAMIENTO DE LOS FONDOS}

Sí creemos que los fondo de inversión van a desarrollar el mercado de valores porque es otra opción en que un inversionista contribuye a dar mayor dinamismo en el mercado porque si se ve la cifra de valores de este año comparada con las del año pasado ve que hay un estancamiento y decrecimiento de las operaciones que hay por bolsa, por lo que creemos que los fondos van a venir a aumentar ese dinamismo y al mismo tiempo un dinamismo en la economía y en los sectores productivos del país porque hay proyectos que se pueden desarrollar a Una de las cosas que hoy en día se tiene es que, hay personas que tal vez no tienen un ahorro, pero ve la figura del fondo de inversión, talvez no la entiende mucho pero ve que existe una posibilidad de invertir. través de los fondos de inversión. 